\title{
Metered-Dose Inhaler With Spacer Instead of Nebulizer During the Outbreak of Severe Acute Respiratory Syndrome in Singapore
}

\author{
See Meng Khoo MBBS MMed, Lian Kheng Tan RN, Nora Said RN, and T K Lim MBBS
}

\begin{abstract}
BACKGROUND: The use of metered-dose inhaler (MDI) with spacer instead of nebulizer may be important during an outbreak of an airborne infection. However, there is a paucity of data on patients' and nurses' abilities and perspectives on MDI with spacer for the treatment of acute airway obstruction during such an outbreak. METHODS: We evaluated 50 consecutive MDI-withspacer treatments administered in the respiratory wards of the National University Hospital of Singapore, and interviewed the patients after each treatment during the outbreak of severe acute respiratory syndrome (SARS). We also conducted interviews with $\mathbf{5 0}$ nurses who had experience in administering bronchodilators via both nebulizer and MDI with spacer. RESULTS: Forty-six patients $(92 \%)$ were able to use MDI with spacer effectively. Sixteen percent of the patients preferred nebulizer over MDI with spacer. Fifty-eight percent of the patients thought MDI with spacer was easier to use than nebulizer, and $34 \%$ thought MDI was as easy to use as nebulizer. Sixteen percent of the patients thought that nebulizer was more effective than MDI with spacer in relieving their symptoms. Ninety-six percent of the nurses preferred nebulizer over MDI with spacer. Fortytwo nurses $(84 \%)$ thought that nebulizer was more effective for treating acute airflow obstruction in the hospital. CONCLUSIONS: In the in-patient setting during an outbreak of an airborne infection, for treatment of acute airflow obstruction, MDI with spacer was acceptable and preferred by a high percentage of patients. However, a high percentage of nurses had misconceptions regarding the efficacy of and patients' ability to use MDI with spacer. Key words: acute airway obstruction, bronchodilators, metered-dose inhaler and spacer, nebulizer, severe acute respiratory syndrome, outbreak, airborne infection. [Respir Care 2009;54(7):855-860. () 2009 Daedalus Enterprises]
\end{abstract}

\section{Introduction}

The nebulizer system of bronchodilator delivery is commonly used for the treatment of acute airflow limitation in hospitalized patients. However, compared to metered-dose inhaler (MDI) with spacer, nebulizer therapy is expensive, uses higher doses of bronchodilator, requires more equipment and equipment maintenance to prevent contamination, and involves more steps in drug preparation and ad-

See Meng Khoo MBBS MMed, Lian Kheng Tan RN, Nora Said RN, T K Lim MBBS are affiliated with the Division of Respiratory and Critical Care Medicine, National University Hospital, Singapore.

The authors have disclosed no conflicts of interest.

Correspondence: See Meng Khoo MBBS MMed, Division of Respiratory and Critical Care Medicine, National University Hospital, Lower Kent Ridge Road, Singapore 119074. E-mail: khoosm@nuh.com.sg. ministration. ${ }^{1}$ We previously found that nebulizer therapy is associated with greater potential for errors in the prescribing and administration. ${ }^{2}$ Importantly, reports from Hong Kong suggested that the use of a nebulizer with a patient who had severe acute respiratory syndrome (SARS) was associated with a major SARS outbreak in the Prince of Wales Hospital. ${ }^{3,4}$ Based on clustering of probable cases

See the Related Editorial on Page 841

of SARS among close contacts of index patients, the main modes of transmission were droplet and direct contact. However, some probable cases of SARS infection contracted inside hospitals did not have direct or close contact (within $1 \mathrm{~m}$ or the range of spread through droplets) with any patient with SARS, which suggested airborne spread through virus-laden aerosols as another likely mode. Aerosol-generating procedures (eg, nebulization) in the hospital 
appear to be associated with a higher potential risk of transmission and have been implicated in nosocomial outbreaks. ${ }^{5}$ These limitations and risks of nebulizer therapy challenge the widely accepted practice of using nebulizer instead of MDI with spacer for in-patient treatment of acute airflow obstruction.

Meta-analysis of randomized controlled studies shows that the delivery of bronchodilators via nebulizer or via MDI is equally effective for treatment of acute air flow obstruction in the emergency department and hospital setting. ${ }^{6}$ However, surveys and audits of practice indicate that nebulizers are preferred by many practitioners. 6,7 This practice is probably based on the belief that, in the hospital setting, many patients are too old or too sick to use the MDI with spacer effectively and the majority of patients prefer MDI with spacer to nebulizer. ${ }^{8}$ There is a paucity of data regarding the feasibility of MDI with spacer for the treatment of acute airflow obstruction in the general unselected in-patient population, and on patients' abilities and perspectives on MDI with spacer in the in-patient setting. ${ }^{9}$ Though nurses' knowledge about and technique with MDI with spacer have been investigated, there are no data on the attitudes of nurses responsible for instructing and supervising the use of MDI with spacer. ${ }^{10,11}$

This study was performed during the SARS outbreak in Singapore. We studied 3 hypotheses: that patients hospitalized with acute airflow obstruction would be able to effectively use MDI with spacer; that MDI with spacer would be acceptable to and preferred by those patients; and that nurses have misconceptions regarding the efficacy of and patients' ability to use MDI with spacer.

\section{Methods}

A series of infection control measures were implemented in all hospitals, following an outbreak of SARS in Singapore in March 2003. A report from Hong Kong ${ }^{3}$ suggested that the use of a jet nebulizer to administer aerosol albuterol in the index patient had probably aggravated the spread of SARS infections. In response to the report, National University Hospital replaced all jet nebulizers with MDIs with spacers for the delivery of bronchodilators in all open wards. Nebulizer was used only when MDI with spacer had been tried and failed to improve patients' symptoms. The respiratory division prepared a guideline on the use of MDI with spacer, which consisted of information on the procedures and doses of bronchodilators. The guideline was distributed to medical and nursing staffs in all general wards (Appendix 1). Volumatic spacers (Allen \& Hanburys/GlaxoSmithKline, Uxbridge, United Kingdom) were made available in all acute medical wards. The urgency to contain the SARS outbreak necessitated an abrupt implementation of the MDI with spacer protocol without formal training sessions for the nurses. Two asthma nurses who
Table 1. Subject Characteristics, Diagnoses, and Bronchodilator Doses

\begin{tabular}{lc}
\hline \hline Age (mean and range y) & $63(18-98)$ \\
Sex (male/female) & $32 / 18$ \\
Diagnosis & \\
$\quad$ Asthma & 21 \\
COPD & 29 \\
Mean doses of nebulized bronchodilators* & \\
All patients & 1.24 \\
$\quad$ Salbutamol (mg) & 238 \\
$\quad$ Ipratropium bromide $(\mu \mathrm{g})$ & \\
Chronic obstructive pulmonary disease & 1.21 \\
$\quad$ Salbutamol (mg) & 240 \\
$\quad$ Ipratropium bromide $(\mu \mathrm{g})$ & \\
Asthma & 1.28 \\
$\quad$ Salbutamol (mg) & 235 \\
$\quad$ Ipratropium bromide $(\mu \mathrm{g})$ &
\end{tabular}

* The 4 patients who failed treatment via metered-dose inhaler with spacer are not included. $\mathrm{COPD}=$ chronic obstructive pulmonary disease

were proficient with the use of MDI with spacer were assigned the duty of providing advice and demonstration of technique to the ward nurses on request. The study was approved by institutional ethics committee.

We evaluated 50 consecutive MDI-with-spacer treatments in the respiratory wards. The patients were interviewed immediately after receiving MDI-with-spacer treatment on the first day of admission to the ward. During each treatment, at the bedside, the nurse instructed the patient on the use of MDI with spacer. Patients with suspected pneumonia were admitted to the isolation wards because of the SARS outbreak and were excluded from the study. We also interviewed 50 nurses who were working in the respiratory ward during the SARS outbreak and who had experience administering bronchodilators via both nebulizer and MDI with spacer. Nurses were interviewed individually after they administered MDI-with-spacer treatments. Appendix 2 shows the interview questions. Effective use of MDI with spacer was defined as satisfactory subjective symptom improvement and, therefore, no need for treatment escalation (bronchodilator via nebulizer, transfer to the intensive care unit, or initiation of mechanical ventilation) and the absence of adverse outcomes. We asked the nurses and patients about their beliefs and preferences regarding nebulizer versus MDI with spacer, their satisfaction with the effectiveness of MDI with spacer, their assessment of ease of use of MDI with spacer, and about the adequacy of the nurses' instruction on MDI with spacer.

\section{Results}

Table 1 shows the patients' demographics, diagnoses, and nebulized bronchodilator doses. All the subjects had 
Table 2. Patient Interview Responses

\begin{tabular}{lccc}
\hline \hline & $\begin{array}{c}\text { Overall } \\
\text { Preference } \\
n(\%)\end{array}$ & $\begin{array}{c}\text { Which Is } \\
\text { More Effective? } \\
n(\%)\end{array}$ & $\begin{array}{c}\text { Which Is } \\
\text { Easier to Use? } \\
n(\%)\end{array}$ \\
\hline Nebulizer & $8(16)$ & $8(16)$ & $4(8)$ \\
MDI with spacer & $25(50)$ & $23(46)$ & $29(58)$ \\
No preference/same & $17(34)$ & $19(38)$ & $17(34)$ \\
MDI = metered-dose inhaler & & & \\
\hline
\end{tabular}

had at least one previous hospital admission in which they received nebulizer therapy. All patients received prednisolone $30 \mathrm{mg}$ per day for 7 days.

Forty-six patients (92\%) were able to use MDI with spacer effectively to achieve satisfactory symptomatic relief. Treatment failure was observed in 4 patients (8\%). Two patients had asthma (ages $49 \mathrm{y}$ and $55 \mathrm{y}$ ) and 2 had COPD (ages $72 \mathrm{y}$ and $74 \mathrm{y}$ ). In all 4 patients, nebulizers were used because of the lack of subjective symptomatic relief after the use of MDI with spacer.

\section{Patient Interview Responses}

Table 2 summarizes the patient-interview responses. Eight patients (16\%) preferred nebulizer over MDI with spacer. Twenty-nine patients (58\%) thought MDI with spacer was easier to use, and 17 patients (34\%) thought MDI was equally easy to use.

Eight patients (16\%) thought nebulizer was more effective than MDI with spacer in relieving their symptoms, and the other 41 patients (84\%) thought MDI with spacer was at least as effective as nebulizer in relieving their symptoms. Ninety-six percent of the patients thought the nurse's instructions on MDI-with-spacer were adequate.

\section{Nurse Interview Responses}

Forty-eight nurses (96\%) preferred nebulizer over MDI with spacer and thought that the hospital should return to using nebulizer after the SARS outbreak was under control. Forty-two nurses $(84 \%)$ stated that nebulizer was more effective for treating acute airflow obstruction in the hospital. Forty nurses $(80 \%)$ thought that nebulizer was easier to administer and required less attention and supervision in in-patients. Thirty-two nurses (64\%) thought that elderly patients were more likely to have difficulty with MDI with spacer.

\section{Discussion}

We found that for the treatment of acute airflow obstruction in a general unselected in-patient population dur- ing an outbreak of an airborne infection, MDI with spacer was acceptable and preferred by a majority of the patients. A large majority of our nurses had misconceptions regarding the efficacy of and ability of patients to use MDI with spacer.

Multiple randomized, controlled studies have confirmed that there is no difference in the pulmonary function response between using a nebulizer and using MDI with spacer for administering bronchodilator therapy. ${ }^{6}$ Despite those findings, administration of bronchodilators via nebulizer is generally accepted as standard treatment for patients hospitalized with acute air flow obstruction. The underuse of MDI with spacer may be due to the lack of data on patients' ability to use the device correctly, the preferences of patients for the devices, and the skills and resources needed to properly instruct the patient in the use of the device outside the setting of clinical trials. ${ }^{9}$ In the present study we investigated these issues in a "real world" setting without the artificial patient selection, intensive coaching, and supervision inherent of a clinical trial.

The patients' perspective on the rapid implementation of MDI with spacer use with minimal staff training and additional resources during the outbreak of an airborne infection has not been investigated previously. The implementation of MDI with spacer use in our hospital may have encountered some resistance from patients for several reasons. First, the need for a rapid response to prevent further spread of SARS had resulted in MDI with spacer being introduced with minimal preparation and support. It was implemented immediately after the circulation of a 2-page guideline prepared by the division of respiratory medicine (Appendix 1) and with the support of only 2 asthma nurses familiar with MDI with spacer use. Second, nebulizer was the standard of care prior to the outbreak, and all patients interviewed had had no previous experience with the use of MDI with spacer for treatment of acute dyspnea. However, our results showed that the majority of patients in the study were able to use MDI with spacer effectively (92\%) and thought that the instructions were adequate (96\%). We also found that MDI with spacer was highly acceptable to the patients. Only $16 \%$ of patients preferred nebulizers to MDI with spacer and thought that nebulizer was more effective than MDI with spacer.

The role of nurses in advocating MDI with spacer use may be an important determinant of patient acceptance. The perspectives of nurses on MDI with spacer use have also not been investigated previously. The results of this study suggested that majority of nurses $(96 \%)$ preferred nebulizer over MDI with spacer, citing the inferior efficacy of MDI with spacer and inability of patients to use this method effectively as important disadvantages. Sixtyfour percent thought that MDI with spacer was too difficult to use in elderly patients hospitalized with acute air flow obstruction. Misconceptions about the efficacy of 
MDI with spacer and the ability of patients to use this device may contribute to sluggish adoption of MDI with spacer use and highlights the importance of educational programs to enhance familiarity with the efficacy and cost effectiveness of MDI with spacer use. The use of MDI with spacer was discontinued in this hospital after the control of the SARS outbreak.

In the present study the mean cumulative doses of albuterol and ipratropium bromide required to achieve satisfactory subjective relief of airway obstruction were $1.24 \mathrm{mg}$ and $238 \mu \mathrm{g}$, respectively. This is consistent with the finding of a previous study, that the majority of patients require less than $2.4 \mathrm{mg}$ of albuterol to achieve sufficient therapeutic response..$^{12}$ Ipratropium bromide was added to the regimen because previous studies indicated greater benefit from dual therapy in patients with more severe air flow obstruction. ${ }^{13}$

Limitations of the present study include the lack of objective measurements of lung function, the unique setting of the study (during the outbreak of SARS), and the fact that patients with suspected pneumonia were excluded from the study. These factors may limit its generalization to other clinical situations. However, as the primary aim of this study is the real-world acceptability of MDI with spacer use, objective lung-function response may not have direct relevance. It is also our impression that patients enrolled in the study are representative of the population admitted to general hospitals with severe acute airways obstruction.

The demonstrated acceptability of MDI with spacer use in our study has important implications for the process of translating the proven efficacy of MDI with spacer from clinical trials into real-world effectiveness. This is especially important when an outbreak of airborne infection necessitates the use of a mode of bronchodilator delivery other than nebulizer. The recent outbreak of SARS, the ongoing outbreak of avian influenza in Asia, the potential risk of nebulizer in transmitting airborne infection, together with the long list of advantages associated with MDI with spacer use have made a compelling case for a more widespread use of MDI with spacer in the in-patient setting.

\section{Conclusions}

In conclusion, this study demonstrated that the use of MDI with spacer for treatment of acute air flow obstruc- tion in an in-patient population during an outbreak of airborne infection is acceptable and preferred by a majority of patients. However, a substantial proportion of nurses have misconceptions regarding the efficacy and the ability of patients to use MDI with spacer. Our data suggests that MDI with spacer is acceptable as a short-term substitute for nebulizer during an outbreak, but its long-term implementation in regular care may be impeded by nursing opinions.

\section{REFERENCES}

1. Bowton DL, Goldsmith WM, Haponik EF. Substitution of metereddose inhalers for hand-held nebulizers. Success and cost savings in a large, acute-care hospital. Chest 1992;101(2):305-308.

2. Khoo SM, Lim TK. Prescribing and administration of nebulized bronchodilators: a prospective audit in a university hospital. Respirology 2003;8(2):205-207.

3. Lee N, Hui D, Wu A, Chan P, Cameron P, Joynt GM, et al. A major outbreak of severe acute respiratory syndrome in Hong Kong. N Engl J Med 2003;348(20):1986-1994.

4. Tomlinson B, Cockram C. SARS: experience at Prince of Wales Hospital, Hong Kong. Lancet 2003;361(9368):1486-1487.

5. Yu IT, Sung JJ. The epidemiology of the outbreak of severe acute respiratory syndrome (SARS) in Hong Kong - what we do know and what we don't. Epidemiol Infect 2004;132(5):781-786.

6. Turner MO, Patel A, Ginsburg S, FitzGerald JM. Bronchodilator delivery in acute airflow obstruction. A meta-analysis. Arch Intern Med 1997;157(15):1736-1744.

7. Tien I, Dorfman D, Kastner B, Bauchner H. Metered-dose inhaler: the emergency department orphan. Arch Pediatr Adolesc Med 2001; 155(12):1335-1339.

8. Mandelberg A, Chen E, Noviski N, Priel IE. Nebulized wet aerosol treatment in emergency department - is it essential? Comparison with large spacer device for metered-dose inhaler. Chest 1997;112(6): 1501-1505.

9. Dolovich MB, Ahrens RC, Hess DR, Anderson P, Dhand R, Rau JL, et al; American College of Chest Physicians; American College of Asthma, Allergy and Immunology. Device selection and outcomes of aerosol therapy: evidence-based guidelines. Chest 2005;127(1): 335-371.

10. Self TH, Arnold LB, Czosnowski LM, Swanson JM, Swanson H. Inadequate skill of healthcare professionals in using asthma inhalation devices. J Asthma 2007;44(8):593-598.

11. Hanania NA, Wittman R, Kesten S, Chapman KR. Medical personnel's knowledge of and ability to use inhaling devices. Metered-dose inhalers, spacing chambers, and breath-actuated dry powder inhalers. Chest 1994;105(1):111-116.

12. Rodrigo C, Rodrigo G. Therapeutic response patterns to high and cumulative doses of salbutamol in acute severe asthma. Chest 1998; 113(3):593-598.

13. Rodrigo GJ, Rodrigo C. The role of anticholinergics in acute asthma treatment: an evidence-based evaluation. Chest 2002;121(6):19771987. 
Appendix 1

Guideline for Use of Spacer Device for Bronchodilator Therapy

1. There is a recent report that aerosol-generating procedures like jet nebulizer may have facilitated transmission of the etiologic agent of SARS in some cases.

2. To reduce the risk of spread of disease, we recommend the use of metered dose inhaler (MDI) with spacer in replacement of nebulizer in the treatment of acute airflow obstruction (acute asthma, exacerbation of COPD) for suitable patients.

3. There is conclusive evidence that administration of MDI with spacer is clinically equivalent to a nebulizer in patients with moderate to severe airflow obstruction.

4. The diagnosis of SARS should be excluded to the extent possible in the individual patient before the initiation of any inhalational therapy.

5. If bronchodilator treatment is clinically indicated in a SARS suspect, it should be administered via MDI with spacer with all precautions taken by all health care workers

6. DO not delay or deny bronchodilator treatment in patients with acute airways obstruction e.g. Asthma or COPD.

MDI + Spacer protocol

1. Doses of bronchodilators

a. 4 puffs of salbutamol $(100 \mu \mathrm{g} \times 4=400 \mu \mathrm{g})$

b. 4 puffs of ipratropium bromide $(20 \mu \mathrm{g} \times 4=80 \mu \mathrm{g})$

2. Method

a. Load the spacer with 2 puffs at the first time and instruct the patient to have 2-5 tidal breaths for efficient drug delivery (If the patient can cooperate, deep breaths with breath holding is recommended).

b. Repeat the procedure until 8 puffs of bronchodilators have been administered (4 puffs of salbutamol and 4 puffs of ipratropium bromide).

c. Oxygen can be administered concurrently via nasal prongs if required.

d. Patient can self-administer bronchodilator treatment with supervision by staff nurse.

e. Staff nurse or doctor must review patient carefully to ensure adequate response.

3. Frequency

a. Repeat every 10-15 minutes for the first hour until condition is stable.

b. Thereafter, repeat every 1 to 4 hours according to disease severity and clinical response.

4. Patient should be encouraged to use his / her own spacer device if available. Otherwise, spacers will be provided by wards. Each patient is to have his own spacer device, which is not to be used by another patient.

5. All acute medical wards should have Volumatic spacers readily available for emergency use. (Recommendation: 5-10 spacers per ward). 
Appendix 2

Interview Questions

\begin{tabular}{|c|c|}
\hline \multicolumn{2}{|l|}{ Interview with nurses } \\
\hline $\begin{array}{l}\text { 1. Which method of administering } \\
\text { bronchodilators is more effective in treating } \\
\text { patient with airway obstruction? }\end{array}$ & MDI+spacer / wet nebulizer / same \\
\hline $\begin{array}{l}\text { 2. Assuming that both methods of } \\
\text { administration are of equivalent efficacy, do } \\
\text { you prefer to administer bronchodilators via } \\
\text { MDI+spacer or wet nebulizer and why? }\end{array}$ & MDI+spacer / wet nebulizer \\
\hline $\begin{array}{l}\text { 3. What are the major problems or difficulties } \\
\text { associated with the use of MDI+spacer in } \\
\text { ward? }\end{array}$ & $\begin{array}{l}\text { Reasons: convenience, less error, less } \\
\text { time consuming, patient factors, etc. }\end{array}$ \\
\hline $\begin{array}{l}\text { 4. Is there a group of patients that you have } \\
\text { particular difficulties with administering } \\
\text { MDI+spacer? }\end{array}$ & Yes / No \\
\hline $\begin{array}{l}\text { 5. When the SARS outbreak is under control, } \\
\text { do you think the hospital should continue to } \\
\text { replace wet nebulizer with MDI+spacer? }\end{array}$ & If yes, which group of patients? \\
\hline \multicolumn{2}{|l|}{ Interview with patients } \\
\hline $\begin{array}{l}\text { 1. The next time you have an attack, would } \\
\text { you prefer wet nebulizer or MDI+spacer? }\end{array}$ & $\begin{array}{l}\text { Wet nebulizer / MDI+spacer / no } \\
\text { preference }\end{array}$ \\
\hline 2. Which is easier to use? & $\begin{array}{l}\text { Wet nebulizer / MDI+spacer / no } \\
\text { difference }\end{array}$ \\
\hline $\begin{array}{l}\text { 3. Which treatment is better at relieving your } \\
\text { symptoms? }\end{array}$ & Wet nebulizer / MDI+spacer / same \\
\hline $\begin{array}{l}\text { 4. Do you think the instruction and coaching } \\
\text { given by the staff nurse on MDI+spacer use } \\
\text { was adequate? }\end{array}$ & Yes / No \\
\hline
\end{tabular}

